\title{
KOPRODUKTION
}

\section{Vom Klienten zum Bürger}

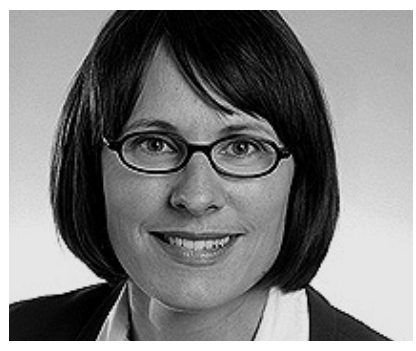

VON BRIGITTE REISER

Dr. Brigitte Reiser ist Diplom-Verwaltungswissenschaftlerin und als Beraterin für Non-Profit-Organisationen tätig. Sie ist spezialisiert auf die Themen Stakeholder-Management und Web 2.0 für gemeinnützige Träger im Sozialsektor.

Internet http://blog.nonprofitsvernetzt.de Internet http://www.stakeholder-management.de
In Deutschland sind viele Bürgerinnen und Bürger sowohl als Nutzer sozialer Dienstleistungen wie als Ehrenamtliche an der Produktion sozialer Dienste beteiligt. Die Planung, Ausgestaltung und Evaluation dieser sozialen Dienstleistungen dagegen liegt oft ausschließlich bei den Sozialunternehmen. Dabei könnten gerade gemeinnützige Organisationen mit dem Konzept der Koproduktion viele Menschen einbinden und so von den Ressourcen ihrer Unterstützer und Partner profitieren.

Koproduktion im Dritten Sektor bedeutet: Dienstleistungen werden durch Netzwerke aus professionellen Anbietern und bürgerschaftlichen Akteuren erbracht - von Nutzern, Angehörigen, Freiwilligen, örtlichen Gemeinschaften usw. Alle am Netzwerk Beteiligten steuern Ressourcen bei. Jedem Stakeholder einer gemeinnützigen Organisation wird zugetraut, dass er zum Koproduzenten werden kann (Bovaird 2007, 847). Sowohl die Leistungserstellung als auch die Planung, das Management und die Evaluation sozialer Dienste können koproduziert werden.

In Deutschland sind viele Bürger in ihrer Rolle als Nutzer sozialer Dienstleistungen und als freiwillige Helfer an der Produktion sozialer Dienste beteiligt - deutlich seltener an deren Planung, Ausgestaltung und Evaluation. »Full user/professional co-production « liegt aber nur dann vor, wenn Dienstleistungen nicht nur gemeinsam erstellt, sondern von Nutzern und der Einrichtung auch gemeinsam geplant und ausgestaltet werden (ebd., 848).

In unserem Land wird die Zivilgesellschaft als praxisorientierte »Helfergesellschaft « (Evers 2009) interpretiert. Die Gestaltungskompetenzen der Bürger, ihre partizipativen Potentiale wenn es um den Diskurs über oder um die Planung von sozialen Dienstleistungen geht, werden unterbewertet und zumeist nicht abgefragt.

Dabei hat der gemeinnützige Sektor nicht nur eine Dienstleistungs- und Interessenvertretungsfunktion. Sondern insbesondere auch die Aufgabe, Menschen in unsere Gesellschaft zu integrieren und ihnen Handlungschancen als Bürger - nicht nur als Klienten - zu verschaffen. Diese demokratiepolitische Funktion von Non-ProfitOrganisationen ist in den letzten Jahrzehnten in den Hintergrund gerückt. Gemeinnützige Träger sehen sich in erster Linie als Dienstleister und nicht als »Enabler ", als Ermöglicher, die Partizipationschancen bieten und Bürgern neue Handlungsräume eröffnen.

Damit vergeben sich gemeinnützige Organisationen die Chance, auf die Ressourcen der Zivilgesellschaft stärker zugreifen zu können - insbesondere auf deren Wissen, das für Non-Profits in Zukunft immer wichtiger werden wird, wenn sie den eigenen Wirkungsgrad und damit auch die eigene Wettbewerbsfähigkeit steigern wollen.

Das Konzept der Koproduktion bindet Bürger in die Planung und Erstellung von Dienstleistungen ein und bietet so die Chance, dass gemeinnützige Organisationen von den Ressourcen ihrer Unterstützer und Partner stärker profitieren können.

Die Koproduktion impliziert einen tiefgreifenden Paradigmenwechsel in gemeinnützigen Organisationen. Die Perspektive der Einrichtungen muss sich verändern. Sie muss weg von der Fixierung auf das eigene System und sich verstärkt zur Umwelt öffnen (vgl. Kasten » Perspektivenwechsel in gemeinnützigen Organisationen «).

\section{Grenzen des Effizienz-Paradigmas im Dritten Sektor}

Der Staat und andere Geldgeber - wie Stiftungen und soziale Investoren - drängen auf mehr Effizienz und Evaluation im NonProfit-Sektor. Gemeinnützige Träger, die um öffentliche und private Mittel konkurrieren, müssen sich diesem institutionellen Druck beugen, wenn sie erfolgreiches Fundraising betreiben wollen. Die Ökonomisierung und Formalisierung des Dritten Sektors wird auch durch die Anstellung von betriebswirtschaftlich und administrativ geschultem Führungspersonal gefördert. Dessen professioneller Antrieb, die Praxis in gemeinnützigen Einrichtungen rationaler, messbarer und effizienter zu machen, unterstützt die Tendenz zur Standardisierung von Leistungen und Wissen im Dritten Sektor (Hwang/Powell 2009). 
Die Standardisierung erschwert Innovationen in gemeinnützigen Einrichtungen. Die Fixierung auf messbare Daten führt zur Vernachlässigung nicht-quantifizierbarer Ressourcen und Ergebnisse. Die Konzentration auf eng definierte Leistungsziele drängt gemeinwesenbezogene Konzepte in den Hintergrund, bei denen Netzwerke - und nicht der binnenorientierte Blick - eine wichtige Rolle spielen. Der Output der gemeinnützigen Einrichtung steht im Mittelpunkt. Welchen Mehrwert die Einrichtung für die Gesellschaft produziert, welche Wirkungen ihre Aktivitäten haben, wird nicht beantwortet. dienstleistungsbezogene Koproduktion (Pestoff 2009, 2007 ff.). Meines Erachtens muss hier noch eine fünfte Dimension hinzugefügt werden: die Koproduktion von Wissen, die Voraussetzung für Innovationen im Dritten Sektor ist (vgl. Kasten »Dimensionen der Koproduktion «).

\section{Koproduktion über das Internet}

Bürger können auch über das Internet an der Planung, Ausgestaltung, Umsetzung und Evaluation von sozialen Dienstleistungen beteiligt werden. Eine wichtige Rolle

\section{"Nicht das rationalisierte System der Dienst-} leistungsproduktion verschafft Non-Profits einen Wettbewerbsvorteil, sondern die gute Qualität ihrer Stakeholder-Beziehungen und

\section{ihrer Netzwerke»}

Die Nachhaltigkeit des gemeinnützigen Handelns geht durch den ökonomisch geprägten Blick verloren, weil man sich am jeweiligen Geschäftsjahr orientiert und längerfristige Horizonte aus der Perspektive geraten. Durch das Effizienz-Paradigma werden die zentrale Steuerung und die Kontrolle in Non-Profit-Organisationen verstärkt. Es existiert nur wenig Raum für die Partizipation von Nutzern und Bürgern. Die Haltung dominiert, Dinge für andere zu tun, statt gemeinsam mit ihnen (Skocpol, zit. nach Hwang/Powell 2009).

Dabei sind es gerade die Beziehungen zu den Stakeholdern, die den Reichtum einer gemeinnützigen Einrichtung ausmachen. Aus den Beziehungen zu den Nutzern, Unterstützern und Bürgern kann eine gemeinnützige Einrichtung die Ressourcen ziehen, die sie benötigt, um innovativ und wirkungsvoll zu sein. Und um mehr Wirtschaftlichkeit zu erzielen, weil mit den Stakeholdern gearbeitet wird und nicht ohne sie und an ihren Bedürfnissen vorbei.

Nicht das rationalisierte System der Dienstleistungsproduktion verschafft NonProfits einen Wettbewerbsvorteil, sondern die gute Qualität ihrer Stakeholder-Beziehungen und der Netzwerke, über die eine Einrichtung verfügt. Auf dieser Überzeugung - dass soziale Beziehungen kein Störfaktor sind, sondern eine zentrale Ressourcenquelle für Non-Profits - basiert das Konzept der Koproduktion (vgl. Boyle/Harris 2009, 11).

Bürger können auf unterschiedliche Weise an der Produktion sozialer Dienstleistungen teilnehmen. Pestoff unterscheidet vier Dimensionen der Koproduktion: die ökonomische, soziale, politische und spielt, wie erwähnt, das Wissen der Bürger, das von Non-Profits immer noch unterbewertet wird. Das Wissen und die Ideen der Bürger könnten helfen, soziale Dienste effektiver auszugestalten. Jedoch muss die $\mathrm{Zi}$ vilgesellschaft die Chance erhalten, ihr Wissen in gemeinnützige Organisationen auch einbringen zu dürfen.

Einige Staaten und Kommunen machen schon vor, wie man Bürgern über das Internet Partizipationschancen bietet, die Innovationen fördern. So stellen beispielsweise die USA (1) und Großbritannien (2) und Städte wie London (3) nicht-personenbezogene Verwaltungsinformationen in Form von Rohdaten zu Tausenden auf Plattformen online und bieten so Bürgern die Chance, das Verwaltungswissen zur Entwicklung von neuen Diensten und Angeboten zu nutzen. Auch an den Non-Profit-Sektor erging der Aufruf, sich hinsichtlich seiner Wissensbestände stärker nach außen hin zu öffnen und Bürger und Nutzer einzuladen, an der Entwicklung von neuem Wissen und innovativen Leistungsangeboten mitzuwirken. (4)

Wie Bürger über das Internet Informationen einbringen und für Non-Profits eine wichtige Wissensquelle sein können, zeigen Web-Kartierungs-Projekte wie GENEVE accessible (5), wo Rollstuhlfahrer hinderliche Barrieren in Genf fotografieren und in Online-Karten eintragen. Oder das WebMapping von Hilfeanfragen im Katastrophenfall - wie beim Chile-Erdbeben -, das von Ushahidi geboten wird (6). Für Hilfsorganisationen werden diese Informationen »von unten ", also von den Bürgern selbst, für die effektive Steuerung eines Hilfseinsatzes immer wichtiger. (7)

\section{Der Nutzen der Koproduktion für gemeinnützige Einrichtungen und Bürger}

Non-Profit-Organisationen können durch die Einbeziehung von Stakeholdern in die Planung und Implementation sozialer Dienstleistungen von den Ressourcen ihrer Nutzer und Unterstützer profitieren. Dabei geht es nicht primär um die monetären Ressourcen der Bürger - obwohl diese Perspektive aufgrund des budgetorientierten Blicks in Non-Profits überwiegt.

Besonders bedeutsam ist das Wissen der Bürger. Gesellschaftliche Probleme sind so komplex, dass sie ohne die Mitwirkung der Nutzer von Dienstleistungen und ihrem sozialen Umfeld sowie dem Wissen und der Mitwirkung der Menschen, die in derselben Gemeinde, im selben Stadtteil leben, nicht gelöst werden können. Probleme verwalten kann man ohne zivilgesellschaftliches Netzwerk Probleme lösen dagegen nicht. Dazu brauchen gemeinnützige Organisationen mehr als professionelle Mitarbeitende, mehr als eine ausreichende Förderung und gute Kontakte in den Staatsapparat - um soziale Probleme zu lösen und damit die eigene Effektivität zu erhöhen brauchen Non-Profits die Zivilgesellschaft.

Der netzwerkorientierte Ansatz der Koproduktion kann gemeinnützige Organisationen und ihre Klientel aus ihren abgesonderten Räumen und Communities befreien, in die sie durch die Spezialisierung, Professionalisierung und Formalisierung des Dritten Sektors geraten sind.

Was wir brauchen, ist die Inklusion betreuungs- und förderungsbedürftiger Gruppen auf breiter Ebene. Und diese Inklusion kann nicht nur als Ziel gemeinnütziger Leistungen definiert werden, sondern muss schon beim Planungs- und Produktionsprozess sozialer Dienste umgesetzt werden, wenn die Inklusion glaubwürdig, nachhaltig und auf Augenhöhe stattfinden soll.

Die Partizipation von Bürgern an der Dienstleistungsproduktion würde das demokratische Profil von sozialen Diensten stärken und ihnen auch auf kommunaler Ebene, wo ihr Hauptarbeitsschwerpunkt liegt, neue Handlungsmöglichkeiten eröffnen: als Akteur, der sich aktiv in die Gestaltung des örtlichen Gemeinwesens einbringt und der Handlungschancen für Bürger schafft, - um damit wieder eigene Handlungsräume zu gewinnen und so mehr Effektivität zu erzielen. Und mehr Effizienz - denn kurzfristig benötigen Koproduktionsstrukturen mehr Mittel, aber langfristig sinken Kosten, weil Bedarfe besser ermittelt, abgedeckt und durch Prävention gemeinsam mit den Bürgernetzwerken verhindert werden können.

Der Nutzen der Koproduktion für Bürger liegt in den neu entstehenden Mitwirkungsmöglichkeiten an der Planung und Ausgestaltung sozialer Dienste. Man würde 


\begin{tabular}{|r|r|}
\hline Perspektivenwechsel in gemeinnützigen Organisationen \\
\hline von der dominierenden Binnensicht & $\rightarrow$ zu den Stakeholder-Netzwerken \\
von der Organisation & $\rightarrow$ zu den Beziehungen \\
von der hierarchischen Steuerung & $\rightarrow$ zur Partizipation \\
von der Professionalisierung & $\rightarrow$ zum Wissen der Bürger \\
vom anbieterzentrierten Servicemodell & $\rightarrow$ zu den Bedürfnissen der Nutzer \\
vom Output & $\rightarrow$ zum Outcome \\
vom Paternalismus & $\rightarrow$ zum Empowerment von Bürger- \\
& Nutzern \\
von ungleichen Machtverhältnissen & $\rightarrow$ zu einem Verhältnis auf Augenhöhe. \\
zwischen der Einrichtung und Bürgern & \\
\hline
\end{tabular}

nicht nur als aktiver Helfer gebraucht, der im Alltag der Einrichtung mit anpackt, sondern auch als Bürger, der seine Ideen und Erfahrungen in die Konzeption sozialer Dienstleistungen einbringt.

Staatliche Institutionen sehen die Koproduktion nur aus der Kosteneinspar-Perspektive: Wie viel Geld kann die öffentliche Hand einsparen, wenn Bürger bei der Betreuung und Pflege von Menschen mithelfen? Das demokratische Potential der Koproduktion - die Mitwirkung der Bürger an der Leistungsausgestaltung - wird von staatlicher Seite nicht gefördert. Im Gegenteil: Die Tendenz zur Zentralisierung von Zielvorgaben und Standards im Dritten Sektor durch den Staat - in Kooperation mit den großen Wohlfahrtsverbänden rückt die Partizipation von Bürgern in weite Ferne. Denn deren Chancen zur Mitgestaltung steigen, je dezentralisierter und je weniger standardisiert soziale Dienste erbracht werden (Pestoff 2009, 221 f.).
Der Nutzen der Koproduktion für die professionellen Mitarbeiter von gemeinnützigen Organisationen liegt darin, dass sie ein neues Profil ausbilden können als Enabler, Unterstützer und Koordinator bürgerschaftlicher Partizipation in Non-Profits (Bovaird 2007, 858). Sie bleiben nicht auf ihre konkrete betreuende und helfende Tätigkeit beschränkt, sondern sie geben hier Verantwortung ab, um dafür mehr gemeinwesenbezogene Handlungsspielräume zu gewinnen. Sie profitieren ferner von der Einbeziehung der Bürger in die Programmformulierung von Non-Profits und gewinnen hier durch die Bürgerbeteiligung Partizipationschancen hinzu. Denn die Bürger-Vorschläge und die professionellen Konzepte müssen untereinander abgestimmt werden, was Aushandlungsprozesse fördert und zentralisierte Standards in den Hintergrund rückt. Dies ermöglicht Kreativität und Innovation auch aufseiten der Mitarbeitenden.

\begin{tabular}{|l|l|}
\hline \multicolumn{2}{|c|}{ Dimensionen der Koproduktion } \\
\hline ökonomische Koproduktion & $\begin{array}{l}\text { Bürger bringen monetäre Ressourcen, } \\
\text { Sachmittel oder ihre Zeit ein, um das } \\
\text { Dienstleistungsangebot eines Trägers zu } \\
\\
\text { erhalten und/oder zu verbessern }\end{array}$ \\
\hline soziale Koproduktion & Bürger investieren Zeit und Material, um \\
& das Sozialleben in einer Einrichtung (in \\
& Form von Festen etc.) mitzugestalten \\
& oder um die soziale Rolle einer \\
& Einrichtung in einer Community zu \\
& erfüllen (Teilnahme am \\
& Weihnachtsmarkt, Tag der offenen Tür) \\
\hline politische Koproduktion & Bürger sind an den Entscheidungen über \\
& die Art der anzubietenden \\
& Dienstleistungen und ihre Ausgestaltung \\
& beteiligt. \\
\hline dienstleistungsbezogene Koproduktion & Bürger wirken an der Erstellung der \\
& Dienstleistungen einer Einrichtung mit. \\
& Entweder, indem sie dieselben \\
& Tätigkeiten wie die professionellen \\
& Mitarbeiter übernehmen, oder deren \\
& Tätigkeit durch komplementäre \\
& Aktivitäten ergänzen. \\
\hline wissensbezogene Koproduktion & Bürger bringen ihr Wissen in \\
& Einrichtungen aktiv ein oder werden von \\
den Einrichtungen gezielt um Ideen und \\
& Beiträge gebeten (»crowdsourcing «). \\
\hline
\end{tabular}

Durch die Koproduktion verändern sich die bisher ungleichen Machtverhältnisse zwischen Bürgern und professionellen Mitarbeitern zugunsten der Bürger. Die Beziehung zwischen Profis und den bürgerschaftlichen Akteuren wird gleichgewichtiger.

Die Veränderung der Machtverhältnisse durch Koproduktion darf von professioneller Seite aber nicht als Verteilungskonflikt wahrgenommen werden. Es geht nicht darum, der professionellen Seite durch mehr Bürgerbeteiligung ein Stück vom Kuchen wegzunehmen, sondern durch die stärkere Einbeziehung von Bürgern den » Ressourcen-Kuchen " von Non-Profits und ihren Mitarbeitern insgesamt zu vergrößern.

Die Zukunft liegt nicht in dem polarisierten Denken von "Wir « und »Die Anderen «, sondern die Zukunft liegt im Poolen unterschiedlicher Kompetenzen und Wissensbestände durch die Non-Profit-Organisation und durch Bürger, um gemeinsam einen Mehrwert für die Gesellschaft zu schaffen (vgl. Boyle/Harris 2009, 12).

\section{Anmerkungen}

(1) Internet http://www.data.gov

(2) Internet http://data.gov.uk

(3) Internet http://data.london.gov.uk

(4) Internet http://blog.nonprofits-vernetzt.de/index.php/open-data-und-dergemeinnutzige-sektor

(5) Internet http://megafone.net/GENE$\mathrm{VE} / \mathrm{map}$.php? $\mathrm{r}=1$

(6) Internet http://chile.ushahidi.com

(7) Internet

http://news.bbc.co.uk/2/hi/technology/8406509.stm

\section{Literatur:}

Bovaird, Tony (2007): Beyond Engagement and Participation: User and Community Coproduction of Public Services, in: Public Administration Review, vol. 67, Heft 5, 846-860).

Boyle, David/Harris, Michael (2009): The challenge of Co-Production, London: NESTA, Internet http://www.nesta.org.uk/publications/reports/assets/features/the_challenge_of_co-pro duction.

Evers, Adalbert (2009): Aktivierung von Zivilgesellschaft in der Sozialen Stadt, in: Newsletter Wegweiser Bürgergesellschaft 23/2009 vom 20.11.2009, Internet http://www.buergergesellschaft.de/fileadmin/pdf/gastbeitrag_evers_o9 1120.pdf.

Hwang, Hokyu/Powell, Walter W. (2009): The Rationalization of Charity, in: Administrative Science Quarterly, Juni, 268-298.

Pestoff, Victor (2009): Towards a Paradigm of Democratic Participation, in: Annals of Public and Cooperative Economics, vol. 8o, Heft 2, 197224. 